\title{
Conformal Algebras of 2D Disordered Systems
}

\author{
Victor Gurarie $^{a}$ and Andreas W.W. Ludwig ${ }^{b}$ \\ ${ }^{a}$ Institute for Theoretical Physics, University of California, Santa Barbara CA 93106-4030 \\ ${ }^{b}$ Department of Physics, University of California, Santa Barbara CA 93106
}

(September 11, 2018)

\begin{abstract}
We discuss the structure of $2 \mathrm{D}$ conformal field theories (CFT) at central charge $c=0$ describing critical disordered systems, polymers and percolation. We construct a novel extension of the $c=0$ Virasoro algebra, characterized by a number $b$ measuring the effective number of massless degrees of freedom, and by a logarithmic partner of the stress tensor. It is argued to be present at a generic random critical point, lacking super Kac-Moody, or other higher symmetries, and is a tool to describe and classify such theories. Interestingly, this algebra is not only consistent with, but indeed naturally accommodates in general an underlying global supersymmetry. Polymers and percolation realize this algebra. Unexpectedly, we find that the $c=0$ Kac table of the degenerate fields contains two distinct theories with $b=5 / 6$ and $b=-5 / 8$ which we conjecture to correspond to percolation and polymers respectively. A given Kac-table field can be degenerate only in one of them. Remarkably, we also find this algebra, and thereby an ensuing hidden supersymmetry, realized at general replica-averaged critical points, for which we derive an explicit formula for $b$.
\end{abstract}

Critical behavior in systems with quenched disorder has remained a major challenge in condensed matter physics. This area includes localization transitions of non-interacting electrons, a prominent example being the Integer Quantum Hall Effect plateau (IQHE) transition [1], but also various random statistical mechanics systems. In two dimensions, powerful techniques of Bethe Ansatz or Conformal Field Theory (CFT) should lead to non-perturbative solutions. Unfortunately this has not been the case so far.

Random critical points in two dimensions, when studied by replica or supersymmetry (SUSY) methods, are believed to be described by CFT [2] with central charge $c=0$. In this paper we put forward a general algebra describing supersymmetric disordered systems. It extends the Virasoro $c=0$ algebra, and is characterized by a parameter $b$ similar to and extending the one found previously by one of us [3]. The crucial novel feature is the appearance of logarithms in the conformal symmetry generators themselves. These then proliferate in almost all correlation functions. Simpler disordered systems, including super Kac-Moody current algebras, are rather special cases of this where additional symmetries prevent these logarithmic features from appearing. It is interesting to note that as soon as the logarithmic features that we describe (such as e.g. Eq.(10)) appear in any $c=0$ conformal theory, SUSY can be accommodated automatically.

We show that this algebra is realized in polymers and percolation. Even though a great deal is known about properties of such geometrical models [4 from their respective mapping onto $q \rightarrow 1$ and $n \rightarrow 0$ limits of $q$-state Potts and $O(n)$ spin models, the nature of the CFT at $q=1$ and $n=0$ is not so well understood. Indeed we show that at $q=1$ or $n=0$, where both models become manifestly supersymmetric [5.,6], these theories develop precisely the logarithmic features of the kind described by our formalism. These, apparently, have been unnoticed in the past. Many critical properties of polymers and percolation have been described by $c=0$ Kac-table degenerate operators. We show that the degeneracy condition of any Kac-table operator fixes the value of $b$ of the theory uniquely. We find, quite remarkably, that this allows for only two possible values, $b=5 / 6$ and $b=-5 / 8$, corresponding to two mutually excluding sets of Kac-table primary operators being degenerate. As a consequence, a given $c=0$ Kac table operator is degenerate only for one choice, not the other. Therefore, the form of correlation functions as deduced from the $c=0$ Kac table degeneracy conditions of a particular operator, cannot be all valid simultaneously for polymers and percolation. We suggest that $b=5 / 6$ corresponds to percolation, and $b=-5 / 8$ to polymers. This should be checked numerically. For percolation this can be done using the super spin-chain formulation of Ref. [6]. A recent mapping of the so-called Spin-Quantum Hall Effect (SQHE) transition [7] onto percolation [5] may be indication that the structure we describe is realized in a variety of delocalization transitions in $2 \mathrm{D}$. We believe that the algebra developed in this paper is a tool to describe and classify generic random critical behavior in 2D.

General Theory: We start by considering a generic disordered system where the disorder average can be performed using SUSY [8]. It has been argued in [3] that the stress tensor of such a system is always a member of a SUSY multiplet. The number of fields in this multiplet depends on the symmetry group of the system. Any such theory must at least be invariant under a minimal $U(1 \mid 1)$ SUSY, giving rise to a 4-dimensional multiplet of stress tensors denoted by $T, t, \xi$ and $\bar{\xi}$ in [3]. If the SUSY is larger, as it is for example the case for the SQHE [6] (where it is $S U(2 \mid 1)$ ), this multiplet will contain more fields, but the above four will always be contained therein. It will suffice to consider those. The action of the four $\mathrm{U}(1 \mid 1)$ generators, two bosonic ( $J$ and $j$ ) and two fermionic $(\eta$ and $\bar{\eta}$ ) on the multiplet of stress tensors is is schematically depicted in Fig. 1 . 


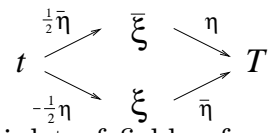

FIG. 1. The multiplet of fields of conformal weight 2 for the group $\mathrm{U}(1 \mid 1)$. The arrows denote acting by the fermionic supersymmetry generators. The stress tensor $T$ is invariant when acted upon by the supersymmetry generators.

In [3] a special role is played by the 'top' field $t$ of this multiplet, whose correlation function with the stress tensor $T$ was argued to be

$$
\langle T(z) t(w)\rangle=\frac{b}{(z-w)^{4}}
$$

where $b$ was a special parameter which counts the number of effective degrees of freedom of the disordered system. Different disordered systems, all having central charge $c=0$, can be distinguished by different values of $b$. It was further argued that the field $t(z)$ together with $T(z)$ should generate an extension of the Virasoro algebra, via their operator product expansion (OPE). However, the most general form of this OPE was not established. Indeed, the OPE proposed in [3] was

$$
T(z) t(0)=\frac{b}{z^{4}}+\frac{2 t(0)}{z^{2}}+\frac{t^{\prime}(0)}{z}+\ldots
$$

Together with the OPE between the stress tensors

$$
T(z) T(0)=\frac{2 T(0)}{z^{2}}+\frac{T^{\prime}(0)}{z}+\ldots
$$

(which simply reflects the fact the central charge of a disordered system is zero) the OPE (2) implies

$$
t(z) t(0)=\frac{2 \kappa T(0)}{z^{2}}+\frac{\kappa T^{\prime}(0)}{z}+\ldots,
$$

where $\kappa$ is some parameter.

The OPEs (2), (3) and (4) are trivially realized in free theories, where disorder is 'switched off', as is readily verified by letting $T=T_{f}+T_{b}$ and $t=T_{f}-T_{b}$. Here $T_{f / b}$ are the stress tensors for the non-interacting fermionic/bosonic parts. Moreover, these OPEs are also realized in certain super Kac-Moody algebras, including [9]. However, these OPEs do not have enough structure to describe generic random systems: by forming suitable linear combination of $T$ and $t$, one simply obtains two commuting Virasoro algebras with equal and opposite central charges. This shows that from the point of view of conformal symmetry (i.e. Virasoro algebras), a super Kac-Moody algebra is isomorphic to a non-random system, albeit with potentially non-trivial factors, replacing the free theories with stress tensors $T_{f / b}$ above.

To rid ourselves from these shortcomings, we now propose the following generalization of the OPE (2), which is still consistent with SUSY, but can accommodate theories with a richer structure,

$$
T(z) t(0)=\frac{b}{z^{4}}+\frac{2 t(0)+\lambda T(0)}{z^{2}}+\frac{t^{\prime}(0)}{z}+\ldots
$$

with some non-vanishing $\lambda$. Indeed, by acting on this OPE with the symmetry generators, following Fig. 1, we can easily show that (3) is consistent with (5). It is probably not possible to modify the OPE (5) any further while keeping it consistent with (3). Since the OPE (2) describes only the simple 'factorized' theories discussed above, while the OPE (5) appears to be the most general OPE we can write for a disordered system invariant under SUSY, we conjecture that the OPE (5) is realized in a majority of SUSY disordered systems, as opposed to special cases such as the ones described by super Kac-Moody algebras or free field theories. Upon rescaling $t \rightarrow t / \lambda$ ( thus: $b \rightarrow b / \lambda$ ) we may set $\lambda=1$ in (5), a choice adopted from now on. Observe also that a shift by $T, t \rightarrow t+\gamma T$ preserves (5).

The OPE (5) harbors many surprises. Its consequences can be investigated with the help of conformal invariance alone, without regard to SUSY. First of all, the form of the OPE (5) implies that $t$ is a logarithmic operator. Logarithmic operators, first introduced in [10], are responsible for the appearance of logarithms in correlation functions directly at criticality, without violating scale or conformal invariance 11]. The reason why the OPE (5) is compatible with, and indeed responsible for, the appearance of logarithms, is because under the action of the dilatation operator $L_{0}$, the field $t$ changes as $L_{0} t=2 t+T$, that is, $\mathrm{T}$ is added to it. This behavior is mimicking the behavior of the logarithms, which, under the change of scale $z \rightarrow \lambda z$, change as $\log (z) \rightarrow \log (\lambda)+\log (z)$. After Ref. [10], many authors worked out a great number of properties of logarithmic operators [12], whose results we will be using. However the logarithmic operator $t$ that we discuss here is special in that it is a logarithmic partner of the stress tensor $T$ itself. It is for this reason that its presence affects the entire theory in a profound way. This distinguishes it in a crucial way from other logarithmic operators discussed elsewhere.

Next, one can show that conformal invariance together with the OPE (3) require that

$$
\begin{aligned}
& t(z) t(0)=-\frac{2 b \log (z)}{z^{4}} \\
& +\frac{t(0)[1-4 \log (z)]-T(0)\left[\log (z)+2 \log ^{2}(z)\right]}{z^{2}}+\ldots
\end{aligned}
$$

up to a certain ambiguity, which we fixed by removing all non-logarithmic terms from the two-point function $\langle t(z) t(0)\rangle$, by shifing with $T$. This OPE replaces the trivial OPE (19). The OPE (6) contains logarithms which is the direct consequence of $t$ being logarithmic.

Since the OPE (6) contains logarithms, any correlation function of the form $\langle t(z) t(w) \ldots\rangle$ will not be singlevalued. If we analytically continue $z$ around $w$, a piece will be added to it. It possible to show that this piece will contain a term precisely of the form $\langle\xi(z) \bar{\xi}(w) \ldots\rangle$ where $\xi$ and $\bar{\xi}$ are (potentially identical) primary operators with conformal weight 2 and a non-vanishing two-point function. In cases of $c=0$ theories with SUSY we clearly 
want to match them with the fermionic fields $\xi$ and $\bar{\xi}$ of the multiplet of Fig. 1. But since our present discussion is more general, not assuming any SUSY, these may be bosonic or fermionic. Let us normalize these operators according to

$$
\langle\xi(z) \bar{\xi}(w)\rangle=\frac{b}{2(z-w)^{4}},
$$

The only OPE between $\xi$ and $\bar{\xi}$ compatible with the correlation function (7), with the OPE's (3), (5) ,(6), and with conformal invariance is

$$
\xi(z) \bar{\xi}(0)=\alpha T(z) T(0)+\frac{b}{2 z^{4}}+\frac{t(0)+T(0) \log (z)}{z^{2}}+\ldots
$$

where a term whose coefficient $\alpha$ is not fixed by conformal invariance may be added. The remaining OPEs between the fields $\xi, \bar{\xi}, T$ and $t$ can also be constructed by conformal invariance. For example,

$$
t(z) \xi(0)=\beta T(z) \xi(0)-T(z) \xi(0) \log z+\frac{\xi^{\prime}(0)}{2 z}+\ldots
$$

Again the coefficient $\beta$ is not fixed by conformal invariance. However, the associativity of the OPE in the threepoint function $\left\langle t\left(z_{1}\right) \xi\left(z_{2}\right) \bar{\xi}\left(z_{3}\right)\right\rangle$ fixes $2 \alpha=\beta$.

Now in a most remarkable way it is possible to show that the OPEs (3), (5), (6), (8), and (9), worked out from conformal invariance alone, using (3) and (5) as a starting point, are automatically covariant under the action of the supergroup, as depicted on Fig. 1. We do need to choose $\xi$ and $\bar{\xi}$ to be fermionic fields and the coefficient $\beta=1 / 4$ in (9), in order to make all these OPEs covariant. But these were the free parameters left for us by conformal invariance. Most of the terms fit into the multiplets by themselves, without any adjustments.

The OPEs (3), (5), (6), (8), and (9) constitute the first and central result of our paper.

One important comment is in order. The OPEs, such as (6) or (8), contain logarithms and are therefore not single-valued. One might think this cannot be true, at least in a SUSY theory, since $t$ and $\xi$ are physical fields, obtained as the " $z z$ " components of the tensors $t_{\mu \nu}$ and $\xi_{\mu \nu}$ introduced in [3]. As such, they have to be singlevalued. As mentioned, the field $t$ (as obtained by supersymmetry), is defined up to a shift $t \rightarrow t+\gamma T$. $\gamma$ can be regularization dependent, and indeed, scale dependent at the critical point, because of $t$ being logarithmic field. This is how the renormalized $t$ can become non singlevalued without any contradictions.

The most interesting consequence of our main result is as follows. Suppose we have a primary field $A$ in our system with conformal weight $h$. The OPE of this field with itself in, say, the holomorphic sector, will contain the contribution of the identity conformal block in the following way,

$$
A(z) A(0)=\frac{1}{z^{2 h}}\left[1+\frac{h}{b}\{t(0)+\log (z) T(0)\} z^{2}+\ldots\right]
$$

that is, it will contain logarithms. As a consequence, the conformal block involving two such fields will contain logarithms as well, as long as the field's conformal weight $h \neq 0$. This can be used as a test if a given disordered system realizes the OPEs with logarithmic $t$ (see the examples below).

Moreover, the above logic can be reversed. Once a given system exhibits the OPE (10), we can argue that it is supersymmetric. Indeed, once a primary operator obeys (10), the field $t$ appears which has to obey (5). Once $t$ obeys (5), the rest of the OPEs such as (6), (8), (9) follow from conformal invariance alone. Once these OPEs appear, they are, as mentioned above, automatically covariant under the SUSY action defined above!

Percolation/Polymers: This ends our general discussion, and we will now consider polymers and percolation (SQHE [6]) as particular examples. Quite remarkably, we will see that they realize the OPE derived above with a logarithmic $t$.

Consider for example the two-point function of the (bulk) energy operator $\epsilon$ in percolation of conformal weight $5 / 8$, in the presence of a conformally invariant boundary. This is believed to be one of the degenerate Kac-table operators at $c=0$ [13]. This function corresponds 14] to a holomorphic four-point function without boundary, with operators located at $z_{1}, z_{2}$ and their mirror images at $\bar{z}_{1}, \bar{z}_{2}$, as in Fig. 2.

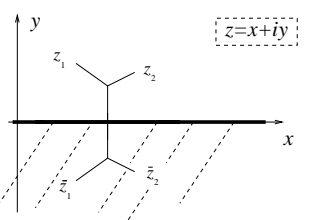

FIG. 2. Points $z_{1}$ and $z_{2}$ in the bulk of the system and their mirror image $\bar{z}_{1}$ and $\bar{z}_{2}$ across the boundary.

The latter can be found by solving the appropriate differential equation, with solution

$$
\begin{aligned}
& \left\langle\epsilon\left(z_{1}\right) \epsilon\left(z_{2}\right)\right\rangle_{\mathrm{bdr}}=\frac{1}{\left|z_{1}-z_{2}\right|^{\frac{5}{2}}(1-x)^{\frac{5}{4}}} \times \\
& {\left[(1-x)^{2} F\left(-\frac{1}{2}, \frac{3}{2} ; 3 ; 1-x\right)+C x^{2} F\left(-\frac{1}{2}, \frac{3}{2} ; 3 ; x\right)\right]}
\end{aligned}
$$

where $x=\frac{\left(z_{1}-z_{2}\right)\left(\bar{z}_{1}-\bar{z}_{2}\right)}{\left(z_{1}-\bar{z}_{2}\right)\left(z_{2}-\bar{z}_{1}\right)}, F$ is the hypergeometric function and $C$ depends on the boundary condition. As the points move away from the boundary $(x \rightarrow 0)$, the correlation function must turn into the bulk two-point function, implying

$$
\begin{aligned}
& \frac{(1-x)^{2} F\left(-\frac{1}{2}, \frac{3}{2} ; 3 ; 1-x\right)+C x^{2} F\left(-\frac{1}{2}, \frac{3}{2} ; 3 ; x\right)}{(1-x)^{\frac{5}{4}}} \propto \\
& \propto 1+\frac{15}{32} x^{2} \log (x)+\ldots, \quad x \rightarrow 0
\end{aligned}
$$


independent of $C$. This demonstrates that this boundary two-point function in percolation, which is given by the above (chiral) four-point function, indeed contains the logarithms which are the hallmark of our formalism. The logarithm appearing here is completely consistent with the OPE (10) if $b=5 / 6$. (The function (11) remains single-valued because $x$ is always a negative real number).

We learn two things about percolation from Eq.(11): (i) Since the logarithmic $t$ operator is known to appear, the theory possesses global SUSY. This follows from the above correlation function, computed by exploiting only conformal symmetry. (A manifestly SUSY invariant formulation of percolation was only very recently found [6]). (ii) the number of physical degrees of freedom $b=5 / 6$ !

The (bulk) energy operator $\epsilon$ (Eq. (11)) is itself logarithmic. Indeed, the SUSY formulation of Ref. [6] showed that it lies in a multiplet isomorphic to that of the stress tensors. Therefore, it also has a logarithmic partner, just like stress energy tensor, whose two-point function contains logarithms, in agreement with results that can be obtained from the 'replica' method of Ref. [17]. However, in spite of the presence of the logarithms in the holomorphic and antiholomorphic sectors one can obtain a single-valued 4-point function in the bulk [18].

For polymers we may consider similarly the two-point function of the bulk energy operator, of conformal weight 1/3, with a boundary as in Eq.(11). This operator is believed to be Kac-degenerate [15]. Explicit calculation as above shows that this function contains again logarithms of the same form as in (12), except that now the coefficient of the log-term is different. Matching this coefficient with (10) yields $b=-5 / 8$. Actually, it appears that all the differential equations following from the $c=0 \mathrm{Kac}$ table degeneracies are consistent with a logarithmic $t$ and its OPEs, if $b$ is chosen to be either $5 / 6$ or $-5 / 8$ depending on which operator we are taking.

This is the second important result of our paper. The $c=0$ Kac table contains operators corresponding to different values of $b$ if we believe that they are degenerate in the standard way and satisfy the standard differential equations. Therefore, such operators cannot be degenerate in the same theory, if we believe in the correlation function of Eq. 111. Take for example percolation: we cannot trust the degeneracy equation for the operator $1 / 3$, because it leads to $b=-5 / 8$ different from the value found from Eq.(11) above, in the same theory. One could try to say that perhaps the same theory contains different fields $t_{b}$ and $t_{b^{\prime}}$ which appear in the OPE (10) for different primary fields with different values of $b$. However, that would violate conformal invariance (to say nothing about supersymmetry). Indeed, in that case the correlation function $\left\langle t_{b} t_{b^{\prime}}\right\rangle$ would be inconsistent with global conformal invariance. So, one should treat the differential equations for the correlation functions of polymers and percolation with care.

Algebraic Formulation: We can set up a completely algebraic way to compute the value of $b$ without solving the differential equations. This is the first step towards a completely algebraic reformulation of the OPEs (3), (5), (6), (8). A study of this algebra and classification of its representations is likely to lead to a description of a quite general class of new disordered critical points.

Concentrate for now on the part of the algebra generated by $T(z)$ and $t(z)$ only. The OPE between $t(z)$ and any primary field $A(w)$ reads (by analogy with (9))

$$
t(z) A(0)=-T(z) A(0) \log (z)+\frac{\frac{1}{2} A^{\prime}(0)}{z}+\ldots,
$$

where ... represent the regular terms in the Laurentexpansion (no logarithms). This allows us to set up a mode expansion of the field $t$ when acting on any primary field $A$ as follows:

$$
l_{n} A(0)=\oint d z z^{n+1}[t(z)+T(z) \log (z)] A(0) .
$$

The argument of the integral is arranged in such a way that it is single-valued as $z$ goes around 0 . This definition leads to the commutation relations between $l_{n}$ and $L_{n}$ in a standard way

$$
\left[L_{n}, l_{m}\right]=\frac{b}{6}\left(n^{3}-n\right) \delta_{n+m, 0}+(n-m) l_{n+m}+n L_{n+m},
$$

This, together with the Virasoro commutation relations allows us to check that the conformal weight $5 / 8$ operator's null vector is primary with respect to both, Virasoro $\left(L_{n}\right)$ and $l_{n}$ generators, only if $b=5 / 6$. This is in agreement with the value of $b$ extracted from the (chiral) four-point function computed earlier in this paper. After checking the operators degenerate down to the 6th level, we discovered that the degeneracy of all the operators of the form $(1, q)$ with $q>2$ which we were able to check required $b=-5 / 8$, while all others we checked required $b=5 / 6$. It is natural to conjecture that this is true of all Kac-table operators, not only those degenerate down to the 6th level, but the mathematical proof of that, based on (15) and the Virasoro algebra, has not yet been obtained.

Replica Theories: Remarkably, we can explicitly identify the logarithmic field $t$ in 'replica' theories, where no SUSY is manifest at the outset. For example in random bond ferromagnets, a multiplicatively renormalizable partner $\tilde{T}^{a}$ of the stress tensor $T$ appears, transforming in the $n-1$ dimensional representation of the replica permutation group [16,17]. $\tilde{T}^{a}$ is primary of dimension $\Delta(n) \rightarrow 2$ as $n, c \rightarrow 0$. We have verified that in this limit the field $\left(\tilde{T}_{a}+\frac{1}{n} T\right)$ is finite and satisfies the OPE's required of $t$, with $-1 / 2 b=\frac{\partial \Delta(n)}{\partial c}{ }_{\mid c=0}$. Hence we arrive at the striking conclusion that the replica treatment of such random systems (allowing for interactions) harbors a hidden SUSY. For polymers and percolation, $n$ is replaced by a corresponding multiplicity, and $\Delta(n)$ naturally coincides with the dimension of $(3,1)$ or $(1,5)$ operators. 
The authors are grateful to I. Gruzberg, N. Read, H. Saleur, and especially to J. Cardy, for useful discussions. One of us (V.G.) is supported by the NSF grant PHY94-07194.

[1] K. von Klitzing et al., Phys. Rev. Lett. 45 (1980) 494.

[2] A. A. Belavin et al., Nucl. Phys. B 241 (1984) 333.

[3] V. Gurarie, Nucl. Phys. B546 (1999) 765

[4] see e.g.; J. Cardy, in 'Les Houches 1994'.

[5] G. Parisi et al., J. de Phys. Lett. 41 (1980) L403

[6] I. Gruzberg et al., Phys. Rev. Lett. 82 (1999) 4524.

[7] T. Senthil et al., Phys. Rev. B 60 (1999) 4245.

[8] K. Efetov, Supersymmetry in Disorder and Chaos (Cambridge Univ. Press, Cambridge, U.K., 1997)

[9] C. Mudry at al., Nucl. Phys. B466 (1996) 383

[10] V. Gurarie, Nucl. Phys. B410 (1993) 535

[11] To our knowledge, the first to notice that logarithms can appear directly at criticality were L. Rozansky at al., Nucl. Phys. B 376 (1992) 461

[12] see e.g.: J.-S. Caux et al., Nucl. Phys. B 466 (1996) 444; I. Kogan et al., Phys. Lett. B375 (1996) 111; Z. Maassarani at al., Nucl. Phys. B489 (1997) 603; I. Kogan at al., Nucl. Phys. B509 (1998) 687

[13] L. Chim et al., Int. J. Mod. Phys. A (1992) 5317.

[14] J. Cardy, Nucl. Phys. B 240 (1984) 514.

[15] A. B. Zamolodchikov, Mod. Phys. Lett. A 6 (1991) 1807.

[16] A.W.W. Ludwig, Nucl. Phys. B330 (1990) 639.

[17] J. Cardy, cond-mat/9911024

[18] V. Gurarie, A. Ludwig, unpublished 